\title{
Moderne Bildgebung in der Dermatologie
}

\author{
Modern Imaging Techniques in Dermatology
}

J. Welzel

Klinik für Dermatologie und Allergologie, Klinikum Augsburg

Korrespondenzadresse

Prof. Dr. med. Julia Welzel

Klinik für Dermatologie

und Allergologie

Klinikum Augsburg

Sauerbruchstraße 6

86179 Augsburg

julia.welzel@klinikum-augsburg. de

\section{Lernziele}

$\nabla$

- Kenntnis der neuen bildgebenden und anderen physikalischen diagnostischen Methoden

- Indikationen für einen Einsatz bildgebender Methoden

- Limitationen der Techniken

- Ablauf und Aufwand der Messungen

- Kenntnis der wichtigsten diagnostischen Merkmale von Hauttumoren

\section{Einleitung}

In der Dermatologie benötigt man zur Diagnostik im Regelfall nur den klinischen Blick und Erfahrung. Die Auflichtmikroskopie ist schon seit vielen Jahren zur Diagnostik von Hauttumoren und Erregern fest etabliert, weil durch die höhere Vergrößerung Details wie Pigmentverteilung, Blutgefäße und sogar größere Einzelzellen sichtbar gemacht werden. Die Sonografie hat sich im Bereich der Lymphknoten- und Gefäßdiagnostik bewährt, aber hochfrequenter Ultraschall spielt aufgrund der zu geringen Auflösung dermaler Veränderungen nur eine untergeordnete Rolle.

In den letzten 10 Jahren sind hochauflösende bildgebende und nichtbildgebende Methoden auf den Markt gekommen, die zahlreiche neue und verbesserte Möglichkeiten zur nichtinvasiven Diagnostik bieten.

\section{Dermatoskopie}

$\nabla$

Die Dermatoskopie ermöglicht eine Lupenbetrachtung der Haut mit 20-60-facher Vergrößerung bis in ca. $0,5 \mathrm{~mm}$ Tiefe. Um die Reflektion der Hautoberfläche zu minimieren, wird die Hautoberfläche an die Optik mit einer Glasplatte und einer Immersionsflüssigkeit angekoppelt oder mit polarisiertem Licht beleuchtet. Es gibt handgehaltene Dermatoskope, Auflichtmikroskope mit Computeranschluss zur Dokumentation und Auswertung sowie Handyskope, die mit
Smartphones genutzt werden können. Zur Verlaufsbeobachtung wird die sequenzielle Videodermatoskopie eingesetzt, mit der Pigmentläsionen über den Zeitverlauf mit hoher Präzision hinsichtlich Veränderungen im Wachstumsverhalten beobachtet werden können.

Die Dermatoskopie wird zur Diagnostik von pigmentierten Hauttumoren eingesetzt [1,2]. Die Pigmentverteilung, aber auch Farbton, Struktur, Randbegrenzung und Symmetrie werden bewertet und geben Hinweise auf die Diagnose.

Die Pigmentstruktur wird maßgeblich von der Körperregion beeinflusst (Gesicht, Akren, Stamm, Schleimhaut). Der Farbton hängt von der Tiefenlokalisation des Pigments ab. Melanozytäre Tumoren weisen typische Pigmentmuster wie Netz, Globuli oder homogenes Pigment auf. Entzündungen führen zu Aufhellungen (Regressionszonen) mit sichtbaren Melanophagen [3]. Pigmentierte Basalzellkarzinome und seborrhoische Keratosen zeigen andere Merkmale wie Teleangiektasien und Pseudohornzysten, die eine Unterscheidung von melanozytären Tumoren ermöglichen. Auch Farbton, -struktur und -verteilung von Angiomen differieren deutlich von melanozytären Läsionen.

Bei nichtpigmentierten Tumoren ist die Dermatoskopie auf die Form und Verteilung der Gefäße angewiesen. Hier gibt es typische Muster: punktartig, kommaartig, Haarnadelgefäße, Baumgefäße... [4].

Ein weiteres Einsatzgebiet der Dermatoskopie stellt die Diagnostik von Erregern und Parasiten dar. Insbesondere Scabiesmilben sind in der Vergrößerung leicht zu diagnostizieren. Auch bei Alopecia areata oder vernarbenden Alopezien kann die Dermatoskopie in der Differenzialdiagnostik und Verlaufskontrolle hilfreich sein. Zusammenfassend ist die Dermatoskopie ein unabdingbares Instrument zur Diagnostik bei verschiedenartigen Hautveränderungen [5].

Die Dermatoskopie ist ein Routineinstrument zum Screening verschiedenartiger Hautveränderungen. 


\section{Sonografie}

\section{$\nabla$}

Die Sonografie spielt in der Dermatologie bei der Lymphknotendiagnostik im Rahmen von Tumorerkrankungen und bei der Gefäßdiagnostik eine große Rolle.

Sie findet in der Regel am liegenden Patienten statt. Der Untersuchungsablauf ist standardisiert. Bei der Mittelfrequenzsonografie werden Geräte mit 7,5-18 MHz eingesetzt. Es gibt verschiedene Anbieter dieser Geräte. Die Transducer müssen mit Ultraschallgel an die Haut angekoppelt werden, um einen Impedanzsprung und somit eine Reflektion des Schalls von der Hautoberfläche zu minimieren. Hochfrequenzultraschallgeräte, die mit Frequenzen von $20-75 \mathrm{MHz}$ arbeiten, brauchen hierzu eine offene oder geschlossene Wasservorlaufstrecke. In Deutschland gibt es nur ein CE-zertifiziertes Hochfrequenz-Gerät, welches an Patienten eingesetzt werden kann, das DUB20/75 (taberna pro medicum, Lüneburg).

Mit der Mittelfrequenzsonografie können Lymphknoten hinsichtlich ihrer Größe und Morphologie beurteilt werden. Hinweise für Malignität sind eine Größe von über $1 \mathrm{~cm}$, ein Längen-BreitenVerhältnis (Solbiati-Index) von unter 2, das heißt eine runde statt ovaläre Form, und ein fehlendes Hiluszeichen. Der Hilus stellt sich bei unauffälligen und reaktiven Lymphknoten echoreich dar, der Randsaum echoarm. In malignen transformierten Lymphknoten zeigen sich zentrale oder asymmetisch periphere echoarme Bereiche $[6,7]$. Zusätzliche Informationen liefert die farbcodierte Duplexsonografie, mit der das Aufzweigen der Lymphknotengefäße (sog. Branching) sichtbar gemacht und beurteilt werden kann [8].

In der Gefäßdiagnostik können mittels farbcodierter Duplexsonografie Klappendefekte bei Varikose ebenso wie Thrombosen dargestellt werden.

Die hochfrequente Sonografie ermöglicht eine Darstellung der Dermis. Unter einem signalreichen Eintrittsecho, welches den Impedanzsprung zwischen der Wasservorlaufstrecke und der Hautoberfläche widerspiegelt, ist die Dermis kräftig echoreich. Je nach Lokalisation ist die Begrenzung zur darunterliegenden echoarmen Subkutis scharf und gerade, wellenförmig oder unscharf. Diese ist durchzogen von hellen, schräg verlaufenden Streifen, den Bindegewebssepten. An manchen Lokalisationen sieht man darunter das Eintrittssignal in den Knochen als kräftig-echogene Linie oder Muskelfaszien als parallele Streifen.

Tumoren, aber auch Ödeme und entzündliche Infiltrate, stellen sich in der Hochfrequenzsonografie als echoarme bis echofreie Bereiche in der echoreichen Dermis dar. Die Auflösung ist nicht hoch genug, um eine Binnendifferenzierung zu ermöglichen ( $\bullet$ Abb.1). Die Hochfrequenzsonografie wird in der Dermatologie somit nur noch zur Dickenbestimmung von Tumoren oder Verlaufsbeobachtung von Bindegewebserkrankun-

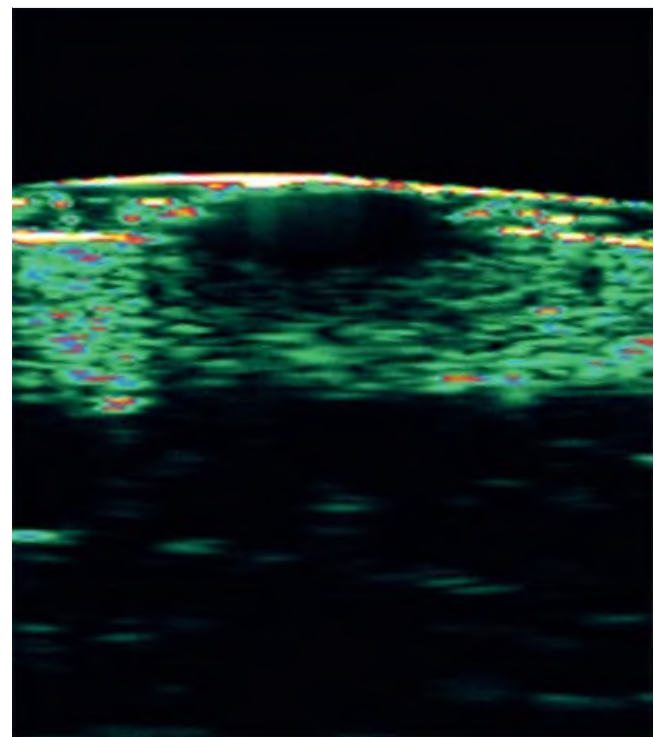

Abb. $120 \mathrm{MHz}$-Sonografie eines dermalen Nävus. Unter der Folie, die die Wasservorlaufstrecke abdichtet, ist das Eintrittssignal in die Haut erkennbar. Der Nävus stellt sich als ovale, echofreie, unscharf begrenzte Raumforderung innerhalb der signalreichen Dermis dar. Eine Binnendifferenzierung ist nicht möglich. $12 \mathrm{~mm} \times 6 \mathrm{~mm}$. gen genutzt, eignet sich aber nicht zur Differenzialdiagnostik [9].

Die hochfrequente Sonografie ist aufgrund der geringen Auflösung nicht zur Diagnostik von Hauttumoren geeignet.

\section{Optische Kohärenztomografie \\ $\nabla$}

Die optische Kohärenztomografie (OCT) ist eine bildgebende Methode, mit der nichtinvasiv zweidimensionale Tiefenschnittbilder der Haut in Echtzeit aufgenommen werden können. Ebenso ist es möglich, horizontale Bilder und dreidimensionale Rekonstruktionen darzustellen. Die Bilder sind einige Millimeter lang, erreichen eine Darstellungstiefe bis in die mittlere Dermis und haben eine Auflösung von unter $10 \mu \mathrm{m}$. Hiermit sind architektonische Veränderungen der Hornschicht, Epidermis und oberflächlichen Dermis darstellbar. Für die konventionelle OCT gibt es derzeit nur ein CE-zertifiziertes Gerät, das VivoSight (Michelson Ltd., Kent, UK). Mit der sogenannten High-definition OCT (HD-OCT) gelingt eine noch höhere Auflösung um $3 \mu \mathrm{m}$, sodass Einzelzellen sichtbar gemacht werden können (Gerät SKINTELL, AGFA, Belgien). Die OCT arbeitet mit Lichtquellen im infraroten Bereich. Die Messungen zweidimensionaler Bilder geschehen in Echtzeit, für dreidimensionale Aufnahmen sind wenige Sekunden nötig. Der Messkopf wird mit einem Abstandshalter, der die Hautoberfläche im Fokus hält, auf die Hautoberfläche aufgesetzt. Bei der HD-OCT sind eine Glasplatte und ein Ultraschallkontaktgel erforderlich, um den großen Messkopf an die Haut anzukoppeln. Die konventionelle OCT kommt mit einem kleinen, flexiblen Handstück ohne Kontaktmedien aus, sodass bei sehr genauen Untersuchungen oberflächlicher 


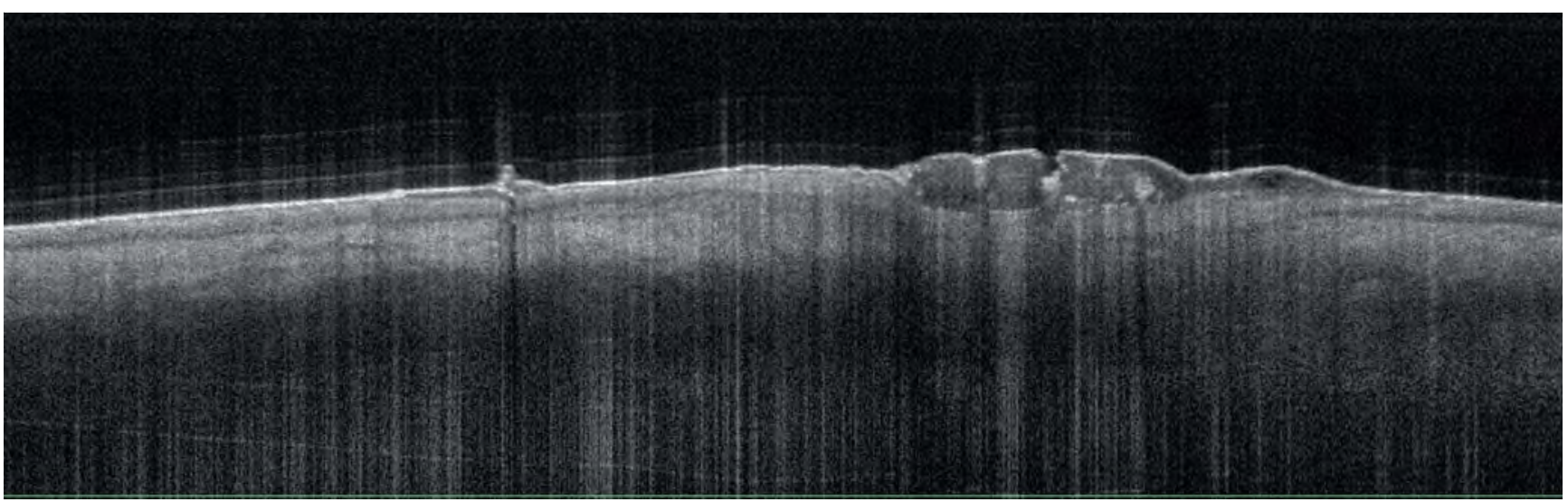

Abb.2 Optische Kohärenztomografie einer aktinischen Keratose. Die Hornschicht ist verdickt und zeigt mehrere signalreichere Schichten. Die dermo-epidermale Junktionszone ist erhalten. $6 \mathrm{~mm} \times 2 \mathrm{~mm}$.

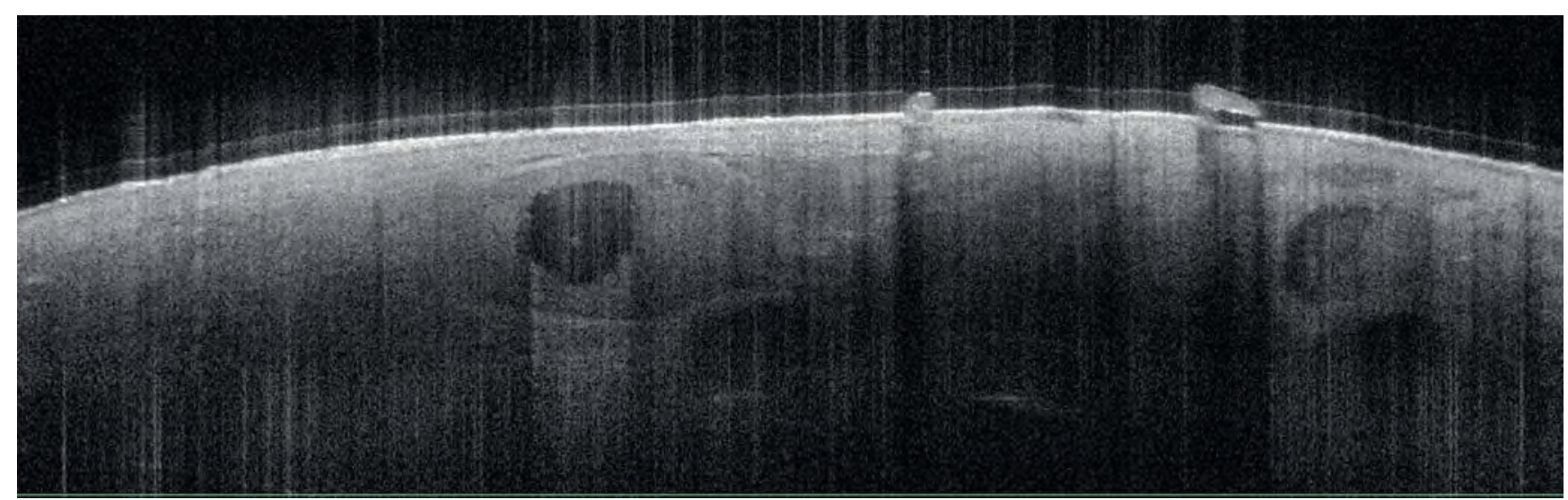

Abb.3 Optische Kohärenztomografie eines solid-zystischen Basalzellkarzinoms. Die Tumorzellverbände stellen sich homogen mit zentralen Zysten, umgeben von einem schmalen dunklen Saum, dar. $6 \mathrm{~mm} \times 2 \mathrm{~mm}$.

Hautschichten keine Artefakte durch beispielsweise eine Hydratation der Hornschicht entstehen können.

Je nach Lokalisation stellt sich die Hornschicht als homogen-signalarme schmale oder breitere Schicht dar, an der Leistenhaut mit spiralförmigen Schweißdrüsenausführungsgängen. Die Epidermis zeigt ebenfalls ein homogenes Signal mit einer scharfen Begrenzung zur Dermis in Form der dermo-epidermalen Junktion. Das Stratum papillare ist signalarm, während das Stratum reticulare der Dermis wieder stärkere, unregelmäßigere Signale zeigt. Blut- und Lymphgefäße sind signalfreie, längliche oder runde Strukturen. Auch Hautadnexe wie Haare, Nägel und Drüsen sind darstellbar $[10,11]$.

Die OCT wird in erster Linie eingesetzt zur Diagnostik epithelialer Hauttumoren. Aktinische Keratosen haben eine kräftige, verbreiterte Hornschicht, meist auch eine Akanthose der Epidermis und eine erhaltene Grenze zur Dermis [12], während invasive Plattenepithelkarzinome diese durchbrechen ( Abb.2). Basalzellkarzinome zeigen sehr charakteristische Veränderungen. Die Epidermis ist meist eher atroph. Fokal zweigen Zapfen von dieser ab. In der Dermis sind ovaläre, homogene, oft zentral zystische Tumorzellinfil- trate sichtbar ( $\bullet$ Abb.3), die eine scharfe Begrenzung durch einen signalarmen Saum aufweisen [13]. OCT ermöglicht zusätzlich zur klinischen und auflichtmikroskopischen Diagnostik eine weitere Erhöhung von Sensitivität und Spezifität in der nichtinvasiven Diagnostik von Basalzellkarzinomen, sodass Biopsien bei eindeutigen Befunden vermieden werden können [14]. Besonders interessant ist die OCT für die Therapieentscheidung und Verlaufskontrolle von Basalzellkarzinomen, da prätherapeutisch die laterale und meist auch die tiefe Begrenzung der Tumoren abgeschätzt werden können $[15,16]$. Bei nichtchirurgischen Behandlungen wie der photodynamischen Therapie oder Imiquimod kann nichtinvasiv kontrolliert werden, ob der Tumor abgeheilt ist $[17,18]$. Vorteile gegenüber Biopsien sind das schnelle Abscannen des gesamten Tumors und die beliebige Wiederholbarkeit von Messungen derselben Region.

Zur sicheren Differenzialdiagnostik von melanozytären Läsionen reicht die Auflösung in der Regel nicht aus. Hier kann OCT ähnlich wie die Hochfrequenzsonografie lediglich zur Dickenbestimmung von Tumoren herangezogen werden [19]. 
Entzündliche Hautveränderungen wie die Psoriasis und degenerative Bindegewebsveränderungen wie Narben können ebenfalls mittels OCT quantifiziert und im Verlauf beobachtet werden.

Zur Erregerdiagnostik wird OCT insbesondere bei Scabies und Onychomykose eingesetzt, während die HD-OCT sogar eine Quantifizierung von Demodexmilben erlaubt.

Eine neue Entwicklung ist die speckle-variance oder dynamische OCT. Hierbei werden durch eine sehr schnelle Messung bewegte Pixel detektiert. Diese korrelieren zu dem Fluss von Blutzellen in Gefäßen, sodass oberflächennahe Blutgefäße, beispielsweise in Hauttumoren, nichtinvasiv dargestellt werden können. Von dieser Technik verspricht man sich zusätzliche Informationen über die Blutgefäßversorgung von Hauttumoren [20], aber es lassen sich beispielsweise auch Effekte von Lasertherapien auf Narben oder topischer Behandlung bei Rosacea quantifizieren.

Haupteinsatzgebiet der optischen Kohärenztomografie ist die Diagnostik und Differenzialdiagnostik epithelialer Tumoren. Sie eignet sich auch zur Verlaufsbeobachtung und Therapiekontrolle.

\section{Konfokale Lasermikroskopie \\ $\nabla$}

Die konfokale Lasermikroskopie (KLM) eröffnet die Möglichkeit, hochauflösend in vivo die Epidermis und das Stratum papillare der Dermis mikroskopisch zu betrachten [22]. Mit einem Laser im nahen Infrarotbereich wird fokussiert eine horizontale Ebene der Haut abgescannt. Eine Lochplatte verhindert, dass störendes Streulicht aus anderen Ebenen die Auflösung verschlechtert. KLM liefert horizontale Schnittbilder der Haut von $500 \mu \mathrm{m} \times 500 \mu \mathrm{m}$ in Echtzeit. Durch eine Lateralverschiebung können sogenannte Blocks innerhalb von Minuten zu zweidimensionalen Übersichtsbildern von bis zu $8 \mathrm{~mm} \times 8 \mathrm{~mm}$ zusammengesetzt werden. Einzelspots können in Form von sogenannten Stacks schrittweise in die Tiefe verschoben werden, sodass man einen dreidimensionalen Eindruck von kleinen Hautausschnitten bekommt. Die Auflösung liegt bei $1-3 \mu \mathrm{m}$ und ermöglicht somit eine Beurteilung der Morphologie von Einzelzellen. Der große Messkopf muss aufgrund der hohen Auflösung, um Bewegungsartefakte weitgehend zu minimieren, mit einem aufgeklebten Magnetring und einer Glasplatte sowie Immersionsöl und Ultraschallgel fest an die Hautoberfläche angekoppelt werden. Da damit nur eine Untersuchung von planen Hautarealen möglich ist, wurde ein flexibles Handstück entwickelt, mit dem schnell Einzelbilder von $1 \mathrm{~mm} \times 1 \mathrm{~mm}$ aufgenommen werden können. Das VivaScope (Mavig, München) ist das einzige kommerziell erhältliche konfokale Lasermikroskop weltweit.

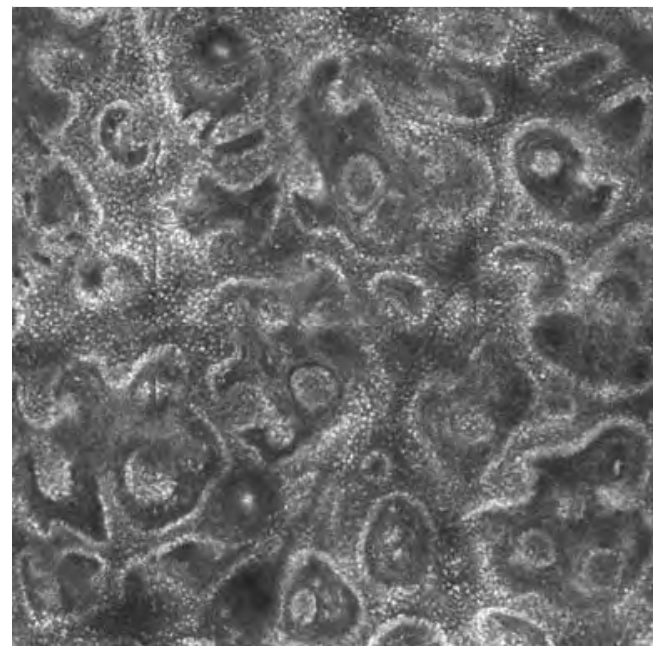

Abb. 4 Konfokale Lasermikroskopie eines Compound-Nävus in Höhe der dermo-epidermalen Junktion. Die Papillen zeigen eine Begrenzung durch regelmäßig angeordnete pigmentierte Keratinozyten. In der oberen Dermis sind einzelne Nävusnester dargestellt. Horizontaler Block, $1 \mathrm{~mm} \times 1 \mathrm{~mm}$.

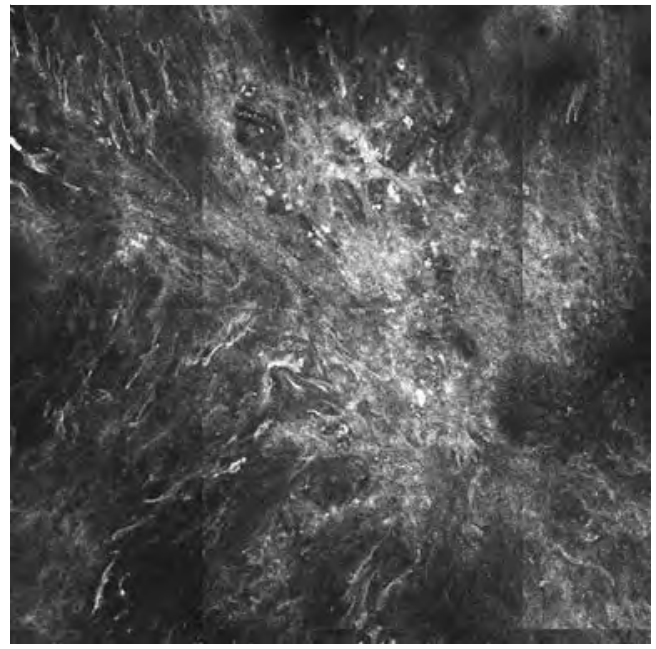

Abb. 5 Konfokale Lasermikroskopie eines malignen Melanoms in Höhe der Epidermis. Reguläre Papillen sind nicht dargestellt. Zahlreiche atypische dendritische oder plumpe Zellen in chaotischer Verteilung durchsetzen die Epidermis. Horizontaler Block, $1 \mathrm{~mm} \times 1 \mathrm{~mm}$.
Die Domäne der KLM ist die Beurteilung melanozytärer Läsionen. Die Technik eignet sich hervorragend, um die Zytologie von Melanozyten und Keratinozyten, aber auch deren Verteilung beurteilen zu können. Nävi zeigen regelmäßige Strukturen mit gleichmäßig pigmentierten Zellen rund um die gut begrenzten dermalen Papillen, in denen sich bei Compound-Nävi dermal gelegene Nester aus monomorphen Zellen abgrenzen lassen ( $\bullet$ Abb.4). Bei Melanomen hingegen ist die reguläre Architektur der Epidermis gestört. Atypische, oft dendritische Einzelzellen findet man bereits in höheren Epidermislagen als Korrelat zur pagetoiden Durchsetzung ( $\bullet$ Abb.5). Die Papillenarchitektur ist ersetzt durch chaotische, oft hell reflektierende atypische Zellen [22].

KLM ist geeignet, Melanome nichtinvasiv von dysplatischen Nävi zu differenzieren, und ist somit eine wertvolle Ergänzung zur Dermatoskopie, um atypische melanozytäre Läsionen einzuschätzen, initiale Melanome früh zu erkennen und andererseits unnötige Biopsien zu vermeiden.

Auch zur Diagnostik von aktinischen Keratosen eignet sich die KLM [23]. Hier ist das typische Wabenmuster der Epidermis gestört durch unre- 
gelmäßig große Keratinozyten. Auch die Parakeratose im Stratum corneum ist sichtbar. Basalzellkarzinome zeigen oberflächliche, oft dunkle Tumorzellverbände mit Randsaum und basaler Palisadenstellung wie in der Histologie [24]. Durch die Echtzeitmessung ist der Blutfluss beurteilbar. In Basalzellkarzinomen sind horizontal verlaufende dilatierte Gefäße mit langsamem Blutfluss sichtbar, oft auch mit großen hellen Zellen, die an der Wand entlangrollen, das sogenannte Leukocyte Trafficking. Zur Beurteilung der Eindringtiefe von Tumoren ist die KLM nur bedingt geeignet, da ab einer Tiefe von $250 \mu \mathrm{m}$ das Bild sehr unscharf wird.

Aufgrund der hohen Auflösung kann die KLM auch eingesetzt werden, um schnell und nichtinvasiv Erreger wie Demodexmilben [25] oder Pilze im Rahmen einer Tinea corporis oder Onychomykose [26] zu diagnostizieren und im Verlauf unter Therapie zu kontrollieren.

Eine Weiterentwicklung stellen Multiwave-Mikroskope dar, bei denen mit mehreren Wellenlängen exogene Fluoreszenzfarbstoffe angeregt werden und dann zusätzlich zum Reflektionsbild auch eine Fluoreszenzfärbung verschiedener Strukturen gelingt. Diese Technik ist insbesondere ex vivo an frisch exzidiertem Gewebe interessant. Zur operativen Schnittrandkontrolle bei Basalzellkarzinomen kann im Sinne einer Schnellschnittdiagnostik das Gewebe innerhalb von Minuten mikroskopiert werden. Hierbei hilft die Fluoreszenzfärbung, beispielsweise mit Acridinorange oder Nilblau, Tumorzellverbände besser vom Stroma abzugrenzen [27].

Die konfokale Lasermikroskopie wird zur Differenzialdiagnostik melanozytärer Tumoren eingesetzt und ermöglicht eine nichtinvasive, hochauflösende Mikroskopie von Nävi und Melanomen.

\section{Multiphotonentomografie}

$\nabla$

Die Multiphotonentomografie arbeitet ähnlich wie die konfokale Lasermikroskopie mit stark fokussiertem Licht. Hierbei gelingt aber durch Zweiphotonenanregung zusätzlich zur Bildgebung durch reflektiertes Licht eine Anregung einer Eigenfluoreszenz von Molekülen wie NADP/NADPH, Melanin, Elastin oder Kollagen. Durch Induktion einer Frequenzverdopplung (,second harmonic generation“) bestimmter Moleküle können insbesondere über das Fasernetzwerk der Dermis weitere Informationen gewonnen werden. Somit ermöglicht die Multiphotonenmikroskopie nicht nur eine hochauflösende In-vivo-Mikroskopie der Haut ähnlich wie die konfokale Lasermikroskopie, sondern liefert zusätzliche funktionelle Informationen über $\mathrm{Ge}$ webe, wodurch eine noch bessere Differenzierung und Auflösung bis in den subzellulären Bereich gelingt. Die Indikationen zum Einsatz der Multiphotonentomografie sind vergleichbar zu denen der KLM, hier allerdings mit dem Fokus auf experimentelle Fragestellungen und Hautalterungsvorgänge [28]. Es gibt bereits ein kommerzielles Gerät zur Diagnostik (DermaInspect, JenLab $\mathrm{GmbH}$, Jena). Insgesamt ist derzeit die Multiphotonentomografie noch sehr aufwändig hinsichtlich der Gerätekosten, und die Messungen müssen in verdunkelter Umgebung stattfinden, sodass ein breiter Einsatz dieser hochinteressanten Technik in der Routinediagnostik derzeit noch nicht stattfindet.

\section{Andere physikalische diagnostische Methoden \\ $\nabla$}

Multispektralanalyse

Die Multispektralanalyse ist eine automatisierte Beleuchtung der Haut mit unterschiedlichen Wellenlängen von 430-950 nm und anschließender bildanalytischer Auswertung der Bilder hinsichtlich Farbverteilung, Symmetrie, Textur und Struktur. Der Algorithmus der Software basiert auf den Daten von ca. 10000 melanozytären Läsionen, die histologisch gesichert wurden, darunter ca. 600 Melanome. Das Gerät Melafind (Melasciences, New York, USA) ist zur Differenzialdiagnostik pigmentierter melanozytärer Läsionen zugelassen, wurde in umfangreichen Zulassungsstudien überprüft [29] und ist so ausgelegt, dass bei geringer Spezifität um 10\% eine möglichst hohe Sensitivität nahe $100 \%$ erzielt wird, um keine Melanome zu übersehen. Es handelt sich nicht um eine bildgebende Methode, sondern die Technik liefert Informationen über die Regularität einer melanozytären Pigmentläsion. Zunächst wurden die Ergebnisse nur als negativ oder positiv ausgegeben. Die neueste Software liefert jetzt Wahrscheinlichkeitsscores für Malignität ( $\bullet$ Abb.6).

Nach einer Eichungsprozedur werden Aufnahmen der Läsion angefertigt. Diese darf keine Narben oder Oberflächenveränderungen wie Ulzeration aufweisen, um die Bilddaten nicht zu verfälschen. Innerhalb kurzer Zeit wird der Score automatisiert angegeben. Nicht geeignet sind akrale, muköse oder subunguale Läsionen, nichtpigmentierte und nichtmelanozytäre Tumoren wie seborrhoische Keratosen oder pigmentierte Basalzellkarzinome.

\section{Impedanzspektroskopie}

Die elektrische Impedanzspektroskopie ist eine Methode, bei der kleine Elektroden sehr oberflächlich in die Haut gepresst werden und der elektrische Widerstand und die Reaktanz (Blindwiderstand) bei verschiedenen Frequenzen zwischen den Elektrodenspitzen in verschiedenen Hauttiefen gemessen werden. Vor der Messung muss die Haut standardisiert angefeuchtet werden. Die Messung dauert wenige Sekunden, wo- 


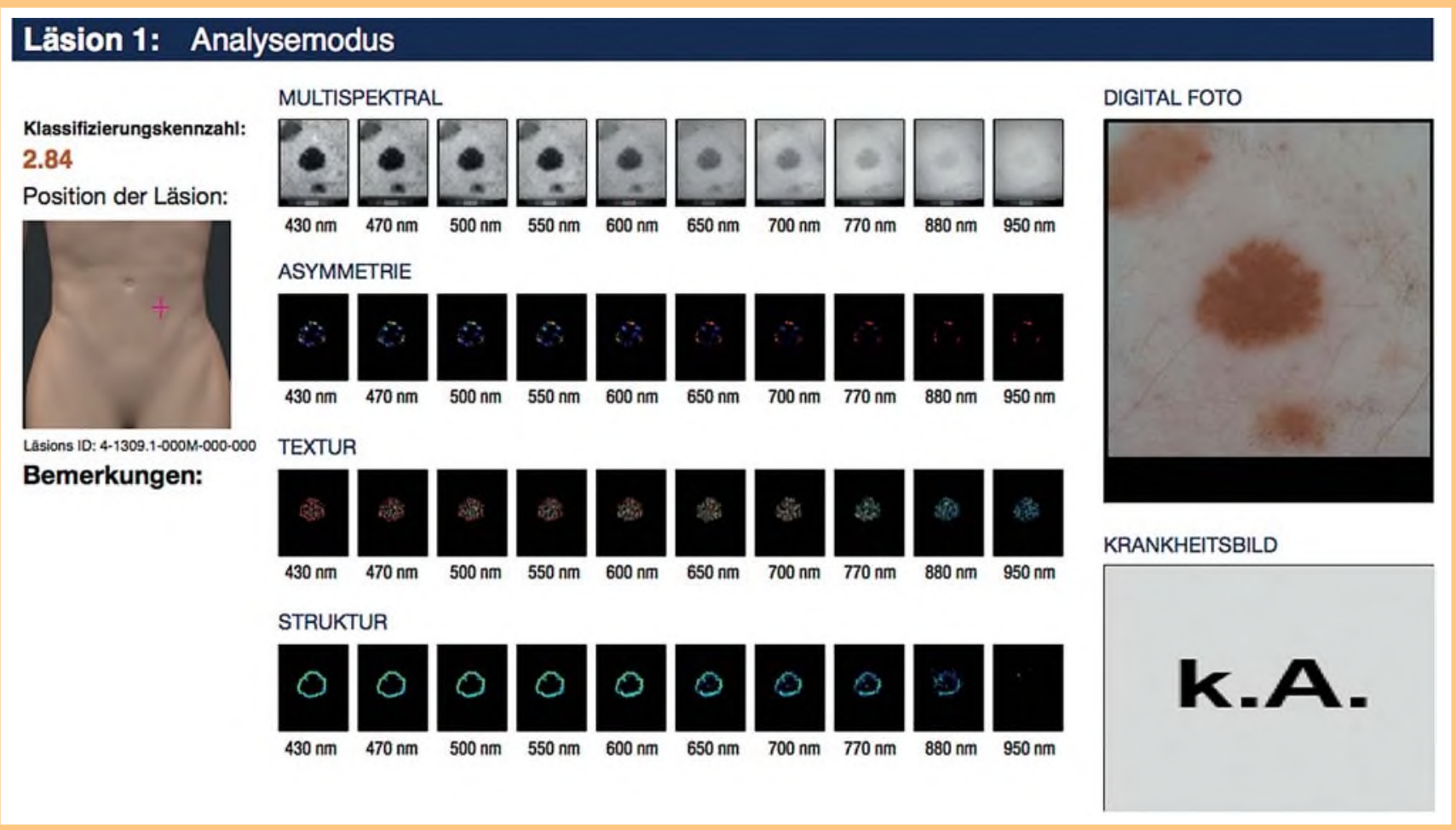

Abb.6 Melafind-Report eines dysplastischen Nävus.

bei immer zusätzlich eine Referenzmessung gesunder benachbarter Haut durchgeführt werden muss. Dargestellt werden die Messkurven und zusätzlich ein automatisierter Score, der die Wahrscheinlichkeit des Vorliegens eines malignen Tumors wiedergibt. Es handelt sich nicht um eine bildgebende Methode, sondern die Stromflüsse spiegeln die Inhomogenität einer Läsion wider (৫ Abb.7).

Das Nevisense System (SciBase AB, Stockholm, Schweden) wurde in mehreren Multicenterstudien überprüft [30] und ist zur Differenzialdiagnostik melanozytärer Läsionen zugelassen. In den Studien wurden ebenfalls nichtmelanozytäre maligne und benigne Tumoren gemessen und ausgewertet. Es hat sich gezeigt, dass für die verschiedenen Entitäten zur Erkennung der Malignität eine sehr hohe Sensitivität nahe $100 \%$ bei niedriger Spezifität von 30-40\% erreicht wird. Die Impedanzspektroskopie ist somit keine Methode zur Diagnostik, sondern zur Beurteilung des Malignitätsrisikos einer Läsion. Bei Oberflächenveränderungen wie Ulzerationen oder Narben und an akralen oder mukosalen Lokalisationen ist die Aussagekraft so eingeschränkt, dass diese Läsionen von der Untersuchung ausgeschlossen werden sollten.

Die nichtbildgebenden physikalischen Methoden der Multispektralmikroskopie und elektrischen Impedanzspektroskopie liefern Scores, die die Malignitätswahrscheinlichkeit angeben. Mit ihnen kann keine Diagnose gestellt werden.

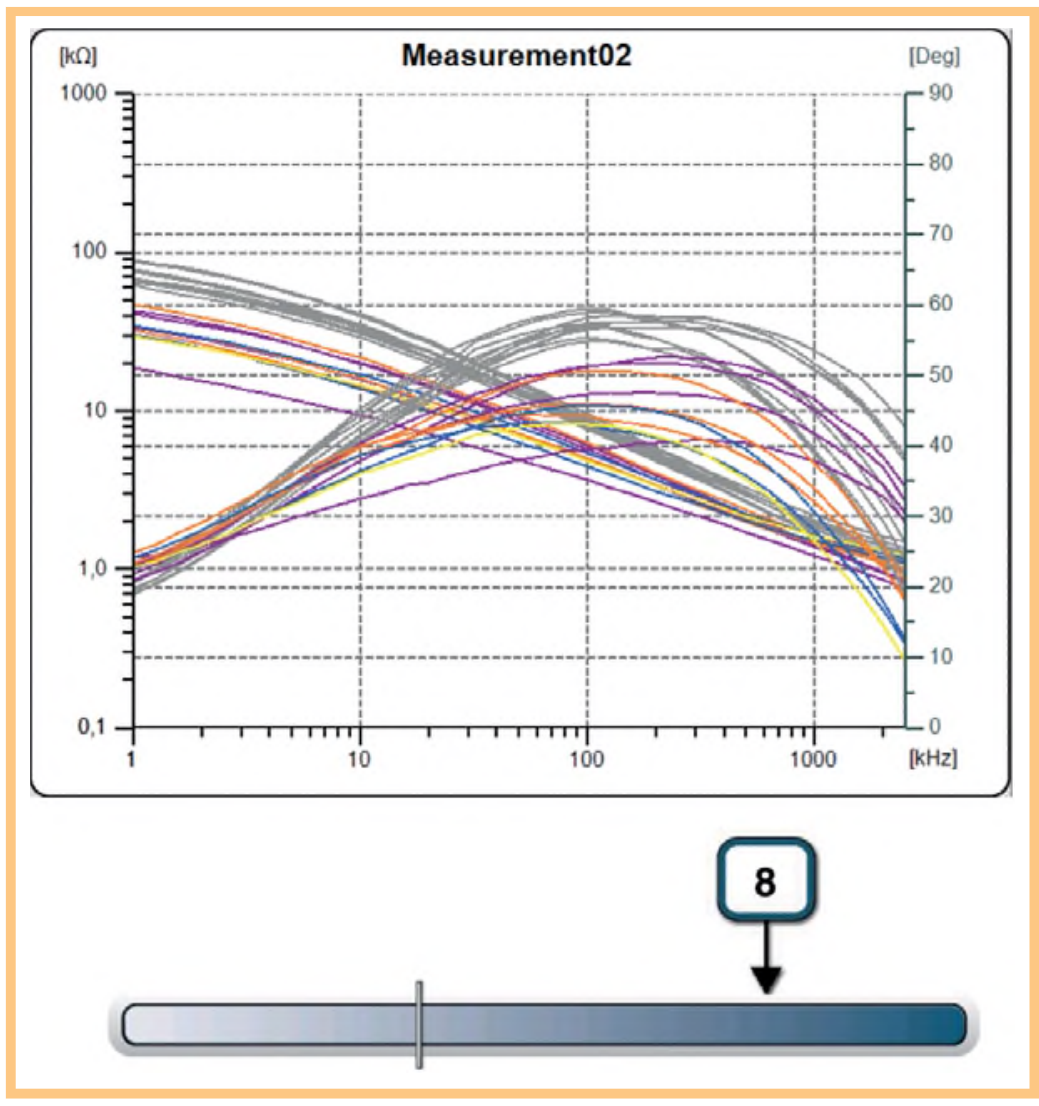

Abb. 7 Elektrische Impedanzspektroskopie eines dysplastischen Nävus. 
Abb. 8 Vergleich der bildgebenden Methoden hinsichtlich Auflösung und Eindringtiefe. $\mathrm{KLM}=$ konfokale Laserdefinition Optische Kohärenztomografie, OCT = Optische Kohärenztomografie, HF-US = hochfrequente $20 \mathrm{MHz}$-Sonografie. mikroskopie, HD-OCT = High-

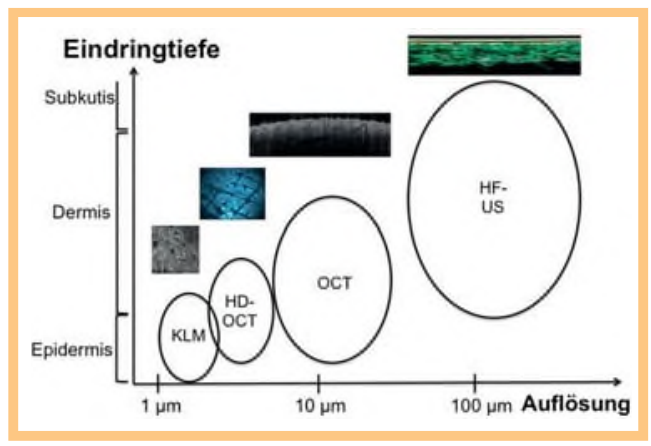

Tab. 1 Indikationen und Spezifikationen der diagnostischen Methoden.

\begin{tabular}{|c|c|c|c|c|}
\hline Methode & Hauptindikation & Auflösung & Eindringtiefe & $\begin{array}{l}\text { Messzeit inkl. } \\
\text { Vorbereitung } \\
\text { und Auswertung }\end{array}$ \\
\hline $\begin{array}{l}\text { Hochfrequente } \\
\text { Sonografie }\end{array}$ & $\begin{array}{l}\text { Tumordicken- } \\
\text { messung }\end{array}$ & $80-200 \mu \mathrm{m}$ & $6 \mathrm{~mm}$ & 5 Minuten \\
\hline $\begin{array}{l}\text { Optische Kohä- } \\
\text { renztomografie }\end{array}$ & $\begin{array}{l}\text { Epitheliale } \\
\text { Tumoren }\end{array}$ & $3-15 \mu \mathrm{m}$ & $1,5 \mathrm{~mm}$ & 2 Minuten \\
\hline $\begin{array}{l}\text { Konfokale Laser- } \\
\text { mikroskopie }\end{array}$ & $\begin{array}{l}\text { Melanozytäre } \\
\text { Tumoren }\end{array}$ & $1-3 \mu \mathrm{m}$ & $200 \mu \mathrm{m}$ & 15 Minuten \\
\hline $\begin{array}{l}\text { Multiphotonen- } \\
\text { tomografie }\end{array}$ & $\begin{array}{l}\text { Diverses/ } \\
\text { Experimentell }\end{array}$ & $1 \mu \mathrm{m}$ & $250 \mu \mathrm{m}$ & 20 Minuten \\
\hline $\begin{array}{l}\text { Multispektral- } \\
\text { analyse }\end{array}$ & $\begin{array}{l}\text { Differenzialdia- } \\
\text { gnostik melano- } \\
\text { zytärer Tumoren }\end{array}$ & Scores & & 3 Minuten \\
\hline $\begin{array}{l}\text { Impedanz- } \\
\text { spektroskopie }\end{array}$ & $\begin{array}{l}\text { Differenzialdia- } \\
\text { gnostik melano- } \\
\text { zytärer Tumoren }\end{array}$ & Scores & & 5 Minuten \\
\hline $\begin{array}{l}\text { Ramanspektro- } \\
\text { skopie }\end{array}$ & $\begin{array}{l}\text { Diverses/ } \\
\text { Experimentell }\end{array}$ & Scores & & 3-10 Minuten \\
\hline
\end{tabular}

\section{Ramanspektroskopie}

Mit der Ramanspektroskopie wird die inelastische Streuung von Licht an Molekülen oder Festkörpern erfasst. Das eingestrahlte Laserlicht wird zurückreflektiert. Neben der Wellenlänge des Lasers werden weitere Frequenzen detektiert, die durch Rotationsschwingungs- oder Spin-FlipProzesse generiert werden. Durch Analyse dieser Spektren können selektiv verschiedene Substanzen in der Haut nachgewiesen werden.

Die Ramanspektroskopie wird in der Dermatologie bisher experimentell zum Nachweis von Antioxidantien in der Haut eingesetzt. Auch für Hauttumore gibt es bereits erste Untersuchungen, die ähnlich wie bei den anderen nichtbildgebenden Methoden Scores der Wahrscheinlichkeiten für den Malignitätsgrad angeben. Dazu ist aber unter Umständen in Zukunft auch eine spezifische Diagnostik verschiedener Tumore möglich.

\section{Weitere Methoden \\ $\nabla$}

In ersten klinischen Studien werden derzeit weitere Methoden zur Hautkrebsdiagnostik überprüft.

Die optoakustische Bildgebung nutzt den photoakustischen Effekt, der die Umwandlung von
Lichtenergie in akustische Energie (Schall) mittels thermoelastischer Expansion beschreibt. Nach Beleuchtung erfährt das Gewebe eine Aufwärmung, die zu einer thermoelastischen Expansion führt. Die dadurch hervorgerufene Schallwelle wird von Ultraschalldetektoren aufgenommen und zur Bildgebung genutzt. In einem dreidimensionalen Bild wird die optische Absorption dargestellt, die unter anderem von der Vaskularisierung abhängt und somit beispielsweise Informationen über eine Tumorangiogenese liefert.

Eine weitere für die Melanomdiagnostik potenziell sehr interessante Methode ist die selektive Anregung der Melanin-Autofluoreszenz durch Zwei-Photonen-Exzitation. Hierbei ist es gelungen, durch Laserlicht weitgehend selektiv die geringe Autofluoreszenz von Melanin anzuregen. Es hat sich gezeigt, dass sich die Fluoreszenzspektren von Melanozyten, Nävuszellen und Melanomzellen unterscheiden, was wahrscheinlich auf das unterschiedliche Eumelanin-/Phäomelanin-Verhältnis der Melanosome zurückzuführen ist [31].

\section{Zusammenfassung und Ausblick \\ $\nabla$}

Die Diagnostik von Hautveränderungen ist in den meisten Fällen einfach durch den klinischen Blick möglich. Erheblichen Zusatznutzen bringt die Dermatoskopie, mit der insbesondere Pigmentläsionen, aber auch unpigmentierte Hautveränderungen, Erreger und entzündliche Hautveränderungen mit höherer Sensitivität und Spezifität diagnostiziert werden können und die ein optimales Screeninginstrument ist. Die Sonografie spielt für die Lymphknotendiagnostik im Rahmen des Stagings und der Nachsorge beim Melanom und für die Gefäßdiagnostik im Bereich der Phlebologie eine unabdingbare Rolle. Zur hochauflösenden bildgebenden Diagnostik epithelialer Hauttumoren hat sich die optische Kohärenztomografie etabliert, während die konfokale Lasermikroskopie ihren Schwerpunkt in der Differenzialdiagnostik atypischer melanozytärer Läsionen findet. Unter den nicht-bildgebenden physikalischen diagnostischen Methoden bieten die Multispektralanalyse und die elektrische Impedanzspektroskopie die Möglichkeit, das Risiko für Malignität einer melanozytären Läsion objektiv mit hoher Präzision abzuschätzen. Es gibt weitere neue diagnostische Methoden, deren Stellenwert derzeit noch unklar ist.

Um die nichtinvasive physikalische Diagnostik als Spezialgebiet zu fördern, einen intensiven und regelmäßigen Erfahrungsaustausch zu ermöglichen, durch Forschungs- und Fortbildungsprogramme zur Erhöhung des Wissens- und Erkenntnisstandes auf dem Gebiet der physikalischen Diagnostik in der Dermatologie beizutragen und die internationale wissenschaftliche Zusammenarbeit zu unterstützen, wurde eine 
Arbeitsgemeinschaft physikalische Diagnostik in der Dermatologie (ApDD) gegründet, die den Arbeitsgemeinschaften der Deutschen Dermatologischen Gesellschaft zugeordnet ist.

Die Zukunft der dermatologischen Diagnostik wird sicher in einer Kombination und in einem differenzierten bzw. sequenziellen Einsatz der Methoden liegen, um ihre Vorteile und Nachteile hinsichtlich Auflösung, Tiefendarstellung, Sensitivität und Spezifität und nicht zuletzt Aufwand optimal auszugleichen ( $\bullet$ Abb.8, $\bullet$ Tab. 1). Die Histologie als derzeitiger Goldstandard in der Diagnostik wird nicht ersetzt werden, sondern durch die Methoden ergänzt, um die Anzahl unnötiger Biopsien zu verringern und subklinische Läsionen früher zu erkennen. In manchen Konstellationen ist eine nichtinvasive Methode sogar der Histologie überlegen, wie beispielsweise beim Screening multipler oder flächiger Hautveränderungen oder in der Verlaufskontrolle.

\section{Fazit}

Die neuen Methoden zur nichtinvasiven Diagnostik unterscheiden sich hinsichtlich Auflösung, Eindringtiefe, Indikation und Aussagekraft. Da sie in vielen Konstellationen ergänzende Informationen zu etablierten Techniken wie der Dermatoskopie liefern, werden die Methoden in Zukunft wahrscheinlich miteinander kombiniert zum Einsatz kommen.

\section{Interessenkonflikt}

Prof. Welzel ist Mitglied im Clinical Advisory Board der Fa. Michelson und Partner im EU-Forschungsprojekt ADVANCE (Automatic Detection of VAscular Networks for Cancer Evaluation) Grant Agreement No. 621015.

\section{Literatur}

1 Kreusch J, Rassner G. Auflichtmikroskopie pigmentierter Hauttumoren. Ein Bildatlas. Stuttgart: Thieme; 1991

2 Stolz W, Braun-Falco O, Bilek P. Farbatlas der Dermatoskopie. 3. Aufl. Stuttgart: Thieme; 2004

3 Blum A, Hofmann-Wellenhof R, Marghoob AA et al. Recurrent melanocytic nevi and melanomas in dermoscopy: results of a multicenter study of the International Dermoscopy Society. JAMA Dermatol 2014; 150: 1-11

4 Kreusch J, Koch F. Auflichtmikroskopische Charakterisierung von Gefäßmustern in Hauttumoren. Hautarzt 1996; 47: 264-272

5 Lallas A, Zalaudek I, Argenziano G et al. Dermoscopy in general dermatology. Dermatol Clin 2013; 31: 679-694

6 Blum A, Schmid-Wendtner MH, Mauss-Kiefer V et al. Ultrasound mapping of lymph node and subcutaneous metastases in patients with cutaneous melanoma: results of a prospective multicenter study. Dermatology 2006; 212: 47-52

7 Voit C, Van Akkooi AC, Schäfer-Hesterberg G et al. Ultrasound morphology criteria predict metastatic disease of the sentinel nodes in patients with melanoma. J Clin Oncol 2010; 28: 847-852
8 Schmid-Wendtner MH, Dill-Müller D, Baumert J et al. Lymph node metastases in patients with cutaneous melanoma: improvements in diagnosis by signal-enhanced color Doppler sonography. Melanoma Res 2004; 50 : 679-682

9 Hoffmann K, Happe M, Schüller S et al. Ranking of $20 \mathrm{MHz}$ sonography of malignant melanoma and pigmented lesions in routine diagnosis. Ultraschall Med 1999; 20 : 104-109

10 Welzel J, Lankenau E, Birngruber R et al. Optical coherence tomography of the human skin. J Am Acad Dermatol 1997; 37: 958-963

11 Mogensen M, Morsy HA, Thrane L et al. Morphology and epidermal thickness of normal skin imaged by optical coherence tomography. Dermatology 2008; 217: 14 20

12 Maier T, Braun-Falco M, Laubender RP et al. Actinic keratosis in the en-face and slice imaging mode of high-definition optical coherence tomography and comparison with histology. Br J Dermatol 2012; 168: 120-128

13 Maier T, Braun-Falco M, Hinz T et al. Morphology of basal cell carcinoma in high definition optical coherence tomography: en-face and slice imaging mode, and comparison with histology. J Eur Acad Dermatol Venereol 2012; 27: e97-104

14 Ulrich M, Maier T, Kurzen H et al. Multicentre study evaluating the sensitivity and specificity of optical coherence tomography for the diagnosis of basal cell carcinoma. $\mathrm{Br}$ J Dermatol 2014; 171: 8

15 Alawi SA, Kuck M, Wahrlich C et al. Optical coherence tomography for presurgical margin assessment of nonmelanoma skin cancer - a practical approach. Exp Dermatol 2013; 22: 547-551

16 Hinz T, Ehler LK, Hornung T et al. Preoperative characterization of basal cell carcinoma comparing tumour thickness measurement by optical coherence tomography, $20-\mathrm{MHz}$ ultrasound and histopathology. Acta Derm Venereol 2011; 92: 132-137

17 Themstrup L, Banzhaf CA, Mogensen $M$ et al. Optical Coherence Tomography Imaging of Non-melanoma Skin Cancer Undergoing Photodynamic Therapy Reveals Subclinical Residual Lesions. Photodiagnosis Photodyn Ther 2014; 11: 7-12

18 Banzhaf CA, Themstrup L, Ring HC et al. Optical coherence tomography imaging of non-melanoma skin cancer undergoing imiquimod therapy. Skin Res Technol 2014; 20: 170-176

19 Gambichler T, Schmid-Wendtner MH, Plura I et al. A multicentre pilot study investigating high-definition optical coherence tomography in the differentiation of cutaneous melanoma and melanocytic naevi. J Eur Acad Dermatol Venereol 2015; 29: 537-541

20 De Carvalho N, Ciardo S, Cesinaro AM et al. In vivo microangiography by means of speckle-variance optical coherence tomography (SV-OCT) is able to detect microscopic vascular changes in nevus to melanoma transition. JEADV 2015 [in press]

21 Rajadhyaksha M, Grossman M, Esterowitz D et al. In vivo confocal scanning laser microscopy of human skin: melanin provides strong contrast. J Invest Dermatol 1995; 104: 946 - 952

22 Pellacani G, Guitera P, Longo $C$ et al. The impact of in vivo reflectance confocal microscope for imaging human tissue microscopy for the diagnostic accuracy of melanoma and equivocal melanocytic lesions. J Invest Dermatol 2007; $127: 2759-2765$

23 Ulrich M, Maltusch A, Rius-Diaz F et al. Clinical Applicability of in vivo Reflectance Confocal Microscopy for the Diagnosis of Actinic Keratoses. J Dermatol Surg 2008; 34: $610-619$

24 Nori S, Rius-Dıaz F, Cuevas J et al. Sensitivity and specificity of reflectance-mode confocal microscopy for in vivo diagnosis of basal cell carcinoma: a multicenter study. J Am Acad Dermatol 2004; 51: 923 -930 
25 Rothmund G, Sattler EC, Kaestle $R$ et al. Confocal laser scanning microscopy as a new valuable tool in the diagnosis of onychomycosis - comparison of six diagnostic methods. Mycosis 2013; 56: 47-55

26 Sattler EC, Maier T, Hoffmann VS et al. Noninvasive in vivo detection and quantification of Demodex mites by confocal laser scanning microscopy. Br J Dermatol 2012; 167: $1042-1047$

27 Gareau DS, Karen JK, Dusza SW et al. Sensitivity and specificity for detecting basal cell carcinomas in Mohs excisions with confocal fluorescence mosaicing microscopy. J Biomed Opt 2009; 14: 034012. DOI: 10.1117/1.3130331

28 Kaatz M, König K. Multiphotonenmikroskopie und Invivo-Multiphotonentomographie in der dermatologischen Bildgebung. Hautarzt 2010; 61: 397-409
29 Monheit G, Cognetta AB, Ferris L et al. The performance of MelaFind: a prospective multicenter study. Arch Dermatol 2011; 147: 188-194

30 Malvehy J, Hauschild A, Curiel-Lewandrowski C et al. Clinical performance of the Nevisense system in cutaneous melanoma detection: an international, multicentre, prospective and blinded clinical trial on efficacy and safety. Br J Dermatol 2014; 171: 1099-1107

31 Leupold D, Scholz M, Stankovic G et al. The stepwise twophoton excited melanin fluorescence is a unique diagnostic tool for the detection of malignant transformation in melanocytes. Pigment Cell Melanoma Res 2011; 24: 438-445 


\section{CME-Fragen Moderne Bildgebung in der Dermatologie}

1 Welche Aussage zur Dermatoskopie ist falsch?

A Die Dermatoskopie ermöglicht eine Beurteilung des Pigmentnetzes in melanozytären Läsionen.

B Die Pigmentstruktur von Nävi hängt von der anatomischen Lokalisation ab.

C Scabiesmilben sind zu klein, um mittels Dermatoskopie erkannt zu werden.

D Gefäßmuster sind wichtige Hinweise für die Diagnostik unpigmentierter Tumoren.

E Paraffinöl ist ein geeignetes Kopplungsmedium für die Dermatoskopie.

2 Welche Aussage ist richtig? Die Mittelfrequenzsonografie wird eingesetzt...

A zum Staging von Lymphknoten beim malignen Melanom.

B zur hochauflösenden Diagnostik von Hauttumoren.

C zur Diagnostik von Organmetastasen.

D um Sentinel-Lymphknoten zu finden.

E zur Verlaufskontrolle von Basalzellkarzinomen nach Operation.

3 Welche Aussage zur Hochfrequenzsonografie ist richtig?

A Sie wird zur Differenzialdiagnostik atypischer melanozytärer Läsionen eingesetzt.

B Sie ermöglicht eine präoperative Dickenbestimmung von Melanomen.

C Die Eindringtiefe beträgt $3 \mathrm{~cm}$.

D Nävi zeigen in der Regel ein anderes Schallverhalten als Melanome.

E Die Auflösung ist so hoch, dass das Stratum corneum an der Felderhaut abgrenzbar ist.

4 Welche Aussage ist korrekt? Die optische Kohärenztomografie...

A liefert Horizontalschnitte der Haut mit einer zellulären Auflösung von $1 \mu \mathrm{m}$.

B benötigt zwingend ein Immersionsöl zur Ankopplung an die Haut.

C ist geeignet zur nichtinvasiven Bildgebung von Basalzellkarzinomen.

D kann sicher zwischen Nävi und Melanomen differenzieren.

E kann Strukturen bis ins Fettgewebe hinein darstellen.
5 Welche Aussage zur optischen Kohärenztomografie von Basalzellkarzinomen stimmt nicht?

A Basalzellkarzinome sind bis zu einer Eindringtiefe von $5 \mathrm{~mm}$ detektierbar.

B Basalzellkarzinome können im OCT-Bild hinsichtlich ihrer lateralen Ausdehnung und Dicke vermessen werden.

C Basalzellkarzinome stellen sich als homogene, signalarme Tumorzellverbände mit einem dunklen Randsaum dar.

D OCT ermöglicht eine nichtinvasive Verlaufskontrolle von Basalzellkarzinomen nach Imiquimod-Therapie.

E Oberflächliche Basalzellkarzinome sind im OCT-Bild detektierbar.

6 Welche Aussage zur optischen Kohärenztomografie von aktinischen Keratosen ist korrekt?

A Aktinische Keratosen sind zu dünn, um im OCT-Bild sichtbare Veränderungen zu zeigen.

B Die Keratose verursacht in der Regel einen totalen Signalschatten.

c Aktinische Keratosen zeigen im OCT-Bild ein atypisches Wabenmuster der Keratinozyten.

D Aktinische Keratosen können nicht von invasiven spinozellulären Karzinomen abgegrenzt werden.

E Aktinische Keratosen zeigen im OCT-Bild eine stark verdickte, inhomogene Hornschicht.

Welche Aussage zur konfokalen Lasermikroskopie ist falsch? Die konfokale Lasermikroskopie...

A liefert Horizontalschnitte der Haut mit einer zellulären Auflösung von 1-3um.

B benötigt zwingend ein Immersionsöl zur Ankopplung an die Haut.

C ist geeignet zur nichtinvasiven Bildgebung von oberflächlichen Hauttumoren.

D ermöglicht eine mikroskopische Beurteilung der epidermalen Veränderungen in Nävi und Melanomen.

E kann Strukturen bis in die tiefe Dermis hinein darstellen.

8 Welche Aussage zur konfokalen Lasermikroskopie von melanozytären Läsionen ist falsch?

A Die konfokale Lasermikroskopie ermöglicht eine Dickenmessung von Melanomen.

B Melanozytäre Nävi zeigen eine reguläre Papillenstruktur.

C Bei Compound-Nävi sind in den Papillenspitzen dermale Nester erkennbar.

D Melanome zeigen eine Störung der epidermalen Architektur.

E Bei Melanomen sind atypische Melanozyten auch in höheren Epidermislagen detektierbar. 
9 Welche Aussage ist korrekt? Die Multispektralanalyse ...

A ermöglicht eine Diagnostik von Hauttumoren.

B ist bei ulzerierten melanozytären Läsionen von über $1 \mathrm{~cm}$ Durchmesser anwendbar.

C benötigt eine intensive Schulung zur Interpretation der Bilder.

D liefert Scores, die eine statistische Malignitätswahrscheinlichkeit melanozytärer Läsionen angeben.

E ist geeignet zur Differenzialdiagnostik zwischen aktinischen Keratosen und Basalzellkarzinomen.
10

Welche Aussage zur elektrischen Impedanzspektroskopie ist korrekt?

A Eine Vorbehandlung der Haut ist nicht erforderlich.

B Die Impedanzspektroskopie kann zur Differenzialdiagnostik zwischen Nävi und Melanomen eingesetzt werden.

C Basalzellkarzinome zeigen ein typisches Bild in der Impedanzspektroskopie.

D Seborrhoische Keratosen können von Melanomen sicher unterschieden werden.

E Die Impedanzspektroskopie kann auch bei akralen melanozytären Läsionen angewendet werden. 